\title{
Designing Maps for Bicycling Simulator Studies - three practical Approaches
}

\author{
Andreas Keler ${ }^{\mathrm{a},}$ *, Patrick Malcolm ${ }^{\mathrm{a}}$, Georgios Grigoropoulos ${ }^{\mathrm{a}}$, Klaus Bogenberger ${ }^{\mathrm{a}}$ \\ ${ }^{a}$ Chair of Traffic Engineering and Control, Technical University of Munich, andreas.keler@tum.de, patrick.malcolm@tum.de, \\ george.grigoropoulos@tum.de, klaus.bogenberger@tum.de \\ * Corresponding author
}

\begin{abstract}
Bicycle simulator studies result from attempts of solving various novel problem statements of modern transportation-related research questions. Examples imply the evaluation of novel traffic control strategies for prioritizing urban bicycle traffic, novel bicycle infrastructure (such as bicycle highways) and the interaction and communication of vulnerable road users with automated or autonomous vehicles. As one of classical disciplines of transportation research, namely traffic engineering, and less related to human factors research, automotive research, geography, urban planning or citizen science, we want to point out those bicycle simulator studies design approaches, which are more related to testing novel traffic control strategies for cyclists, experiencing changing traffic-efficiency and -safety-related parameters in ongoing interfaced microscopic traffic flow simulations. We believe that this is a key factor in experiencing various traffic situations and the evaluation of thereof. In this research, we introduce three practical approaches of how to design maps for bicycling simulator studies. This is mainly resulting from manifold practical experiences from already conducted simulator studies beginning from the year 2018.
\end{abstract}

Keywords: Virtual Reality, Scenario Design, Bicycle Simulator, Traffic Simulation, Communication, Simulator Studies, HD Maps

\section{Introduction}

In the last decades, more and more virtual reality (VR) applications appeared, where the vulnerable road user (VRU), which are by definition all pedestrians and cyclists (when not estimating the scale of vulnerability), is depicted as a virtual model in a three-dimensional environment and steered by a test subject (in a physical simulator). The ideas of implementing these experiments go in the direction of recording behavioural information, similar to the aims of car simulator studies, but with a stronger focus on interaction and communication. A selection of these VRU simulators depict simulated urban traffic and allow the test subject to perform implicit and explicit gestures for signalling information on their purposes of movement strategies.

In this work, we suggest, in our opinion, very recent and practical approaches of designing maps for bicycling simulator studies. We describe one approach based on open source software, basically with the solutions CARLA (Pabst 2020), and two approaches with a commercial software, namely SILAB (Keler et al. 2021a) and DYNA4 (Keler et al. 2018). In Addition, we introduce first experiences with simulator studies in Unity3d from the perspective of vulnerable road users. Alongside these design description, we discuss the value of highly(spatially)accurate geodata of transport infrastructure and its depiction via OpenDRIVE standard, which is currently supported by CARLA. Moreover, we reason on the design processes of the conversion workflows and explain whether the present geodata or a simulation network of the same should be used as the base for representing space in VR. This is a crucial point, since we mostly simulate other road users via microscopic traffic flow simulation tools as
SUMO and the simulation network should have, more or less, the same topological relationships of transportinfrastructural elements. Besides this, all designed scenarios can originate from real-world examples of intersections, including signalized and non-signalized crossings, T-junctions and roundabouts, but instead of real relationships of road segments, can be connected by fictional infrastructure. Therefore, we are able to create bicycle simulator studies such as the one pictured in Figure 1 , which is the second bicycle simulator study for the ongoing (publicly-funded) research project @CITY on autonomous driving functions in the city, with 41 scenarios in total.

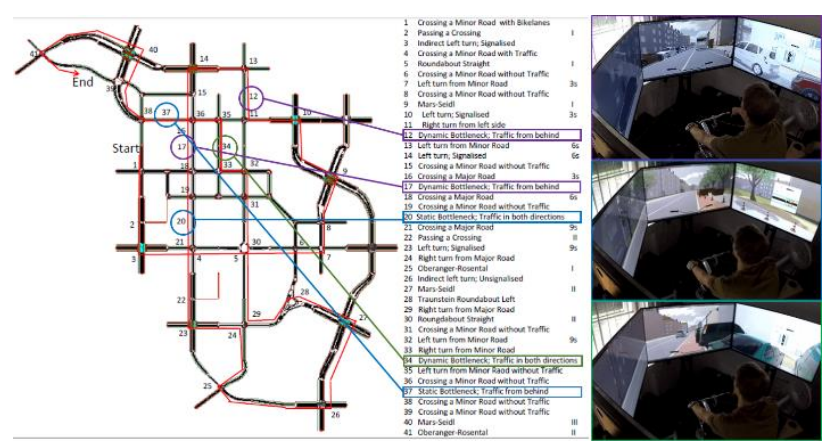

Figure 1. Map view and scenario specifications for the (ongoing, 2021) second bicycle simulator study for the publicly-funded research project @CITY.

On the right of the map view in Figure 1 the specifications are shortly described together with photos of a test subject at the bottleneck scenarios. In general, we use a depth camera of the type Intel ${ }^{\circledR}$ RealSense ${ }^{\mathrm{TM}}$ D435 for obtaining depth information and estimating skeletons or every test subject, as pictured on the left of Figure 2 (camera is 
attached to a tripod). The outcomes of the skeleton estimation are pictured on the right of Figure 2, where a specific hand gesture is being transmitted into the VR animation of the ego cyclist. It is also visible here that the option of wearing VR glasses often comes together with a more careful setup of the hardware and the usage of screens might restrict the visibilities of selected scenarios as for example approaching complex urban intersections.

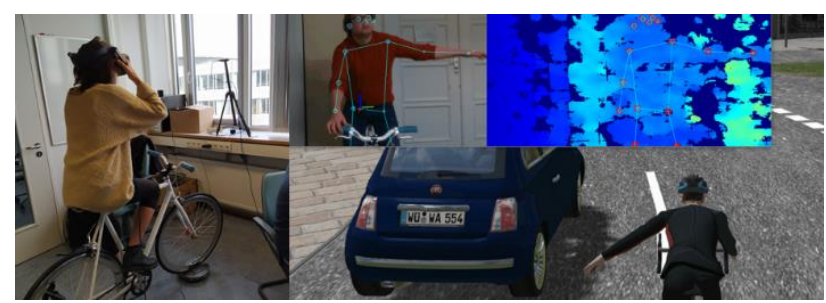

Figure 2. Test subjects being tracked by a depth camera together with VR glasses (left) and TV screens together with depiction of gesture movement of an ego cyclist in the VR environment (right).

As a starting point for designing bicycle simulator scenarios it is possible to extract an OSM file of a certain investigation area, such as pictured in Figure 3, the Williamsburg Bridge. In a subsequent step the OSM extract can be converted via the netconvert tool of SUMO (https://sumo.dlr.de/docs/netconvert.html) into a SUMONet-File serving as a base for the calibration of a (microscopic) traffic flow simulation.

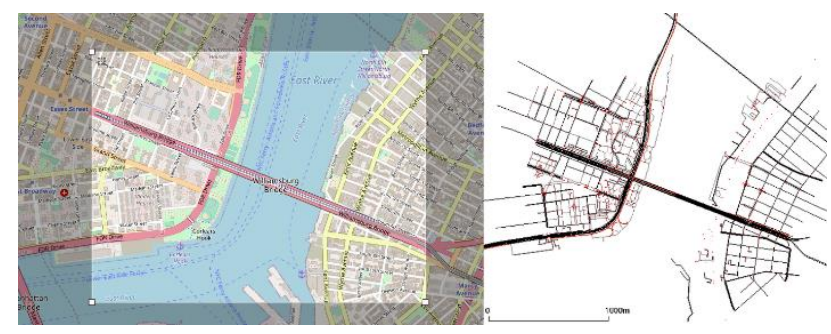

Figure 3. Netconvert example of the Williamsburg Bridge with OSM extract (left) and resulting conversion outcome, a SUMONet-File, (right).

There are multiple options after this conversion step, for the case of DYNA4, it is possible to convert the SUMONet-File further into an OpenDRIVE file, which might be adjusted due to conversion artifacts in the resulting network with open software such as ODDLOT (https://www.hlrs.de/solutions-services/serviceportfolio/visualization/driving-simulator/oddlot/).

Another option would make use of the SumoNetVis python library (https://pypi.org/project/SumoNetVis/) for converting the SUMO network into an OBJ file (Malcolm et al. 2020). Besides these road-segment-related conversions, there are further options of adding building models, and other static objects, which might partially serve as landmarks for the simulator studies itself by means of orientation.

Another topic is adjusting the studies towards controlling traffic light signals or other (partially interacting) road users depending on the respective research questions. One of the previously-conducted studies was for example calibrated with real traffic count and traffic light signals from the city of Munich leading to unpredictable problems of simulator study design and reproducibility, which will be explained in more detail in relation to other bicycle simulator study designs (serving several other purposes).

\section{Overview on Methods - Bike Simulator Study Designs}

In general, we can say that the applications we were and are using for our research purposes are related with an interconnection with a microscopic traffic flow simulations and the experience from an ego perspective. This approach is already well-discovered as shown in Table 1. All of the approaches in Table 1 have an interface between the open microscopic traffic flow simulation software SUMO and commercial software or a free application based on a game engine.

\begin{tabular}{|c|c|c|}
\hline Name or Interface & $\begin{array}{l}\text { Year of First } \\
\text { Application }\end{array}$ & Reference \\
\hline $\begin{array}{l}\text { Coupling SUMO and } \\
\text { SILAB }\end{array}$ & 2018 & $\begin{array}{l}\text { Barthauer and } \\
\text { Hafner } 2018\end{array}$ \\
\hline $\begin{array}{l}\text { SUMO - DYNA4 } \\
\text { Interface }\end{array}$ & 2018 & $\begin{array}{l}\text { Keler et al. } \\
2018\end{array}$ \\
\hline $\begin{array}{l}\text { SUMO } \quad-\quad \text { IPG } \\
\text { CarMaker Interface }\end{array}$ & 2016 & $\begin{array}{l}\text { Kaths and } \\
\text { Krause } 2016\end{array}$ \\
\hline
\end{tabular}

Table 1. Selected Interfaces between SUMO and additional Software from Literature.

\subsection{The DYNA4 Approach}

The DYNA4 approach is addressed in Keler et al. (2018), Keler et al. (2019a, 2019b) and in Malcolm (2019). Similar to the workflow in Figure 3, most map designs rely on real investigation areas or extracts of these from OSM (Keler et al. 2021). Besides recording the test subject trajectories from SUMO, additional data is gathered coming from depth cameras, which is then being used for classifying gestures and matching them to segments of respective trajectory.

As in the study within the project RadOnTime (Keler et al. 2019a), it is possible to trigger traffic light signals and countdown timer displays to guarantee similar conditions for every test subject. Examples of such displays are pictured in Figure 4.

In another bicycle simulator study a calibrated simulation network served for evaluating different implementation options of urban bicycle highways. Based on data coming from traffic observations via video camera devices and of the traffic light signals, it is possible to calibrate a simulation network with real data, for a selected time window (time of the day). Figure 5 pictures this relation with an additional depiction of the VR environment experienced by the respective test subject. Besides changing the geometry of the bike way (bicycle highway lanes), there is a variation in established traffic control 
strategies in the VR environment with and without a prioritization of bicycle traffic.

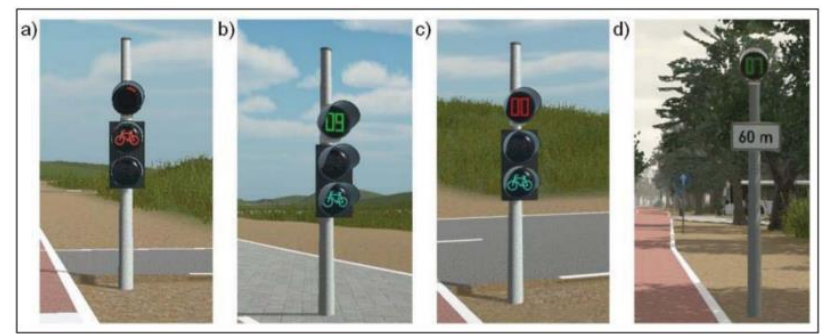

Figure 4. Traffic light and countdown timer display visualizations based on Keler et al. (2019a) and Kaths et al (2021a) used for the simulator study within the project RadOnTime.

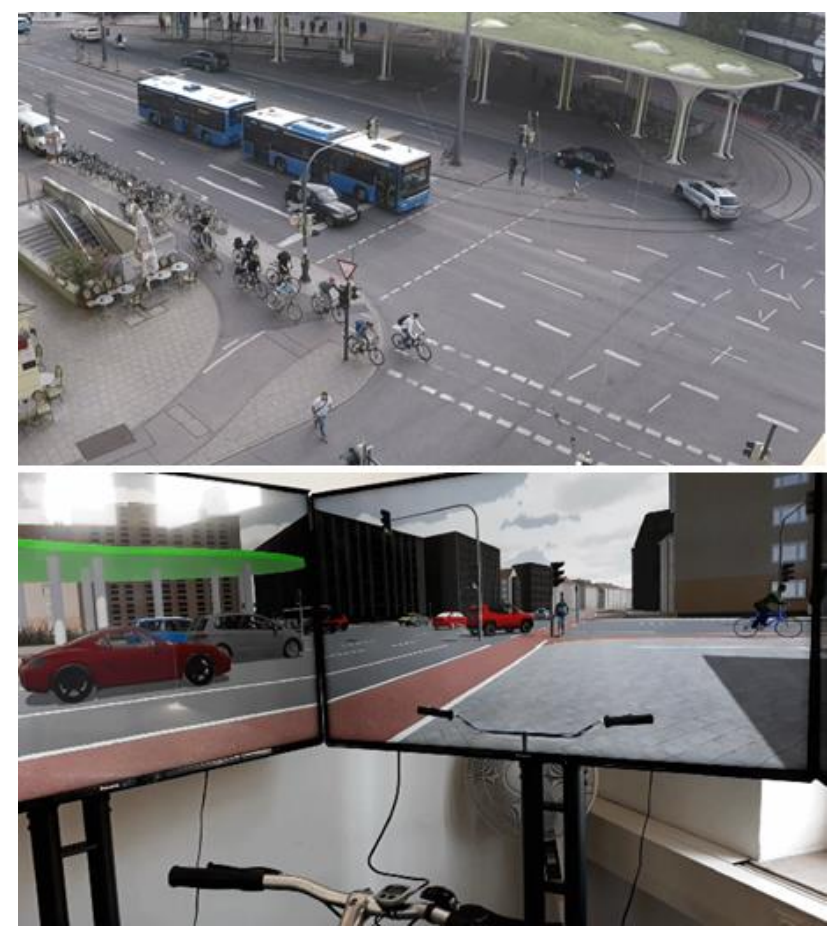

Figure 5. Depiction of a real scene from a 2018 video acquisition at the Münchner Freiheit intersection in Munich, Germany, (upper image) served for extracting mode-dependent traffic count information from totally six observations at the RASCH investigation area (Keler et al. 2018), and (lower image) VR depiction of the interfaced SUMO simulation of the same investigation area based on Keler et al. (2018) and Kaths et al. (2021b).

\subsection{The SILAB Approach}

With the SILAB approach, we build on an already established interconnection between two simulators and exchange one of them as being a cyclist, who is controlling the triggering of the traffic light signal in front of a static bottleneck. Figure 6 pictures a map view of the study area and the different flow points triggering specific events.

The focus here is to gain knowledge on possible interaction and communication between the two test subjects, also supported by the animated gesture movement as pictured in Figure 2.

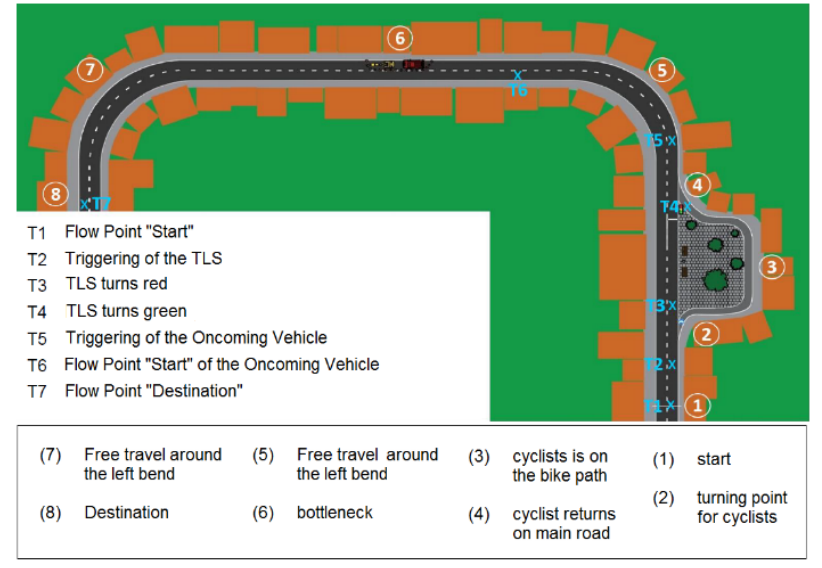

Figure 6. Overview on the static bottleneck scenario used for the interconnected simulator study with ascending numbers representing the cyclist route and flow points triggering events (Tn).

\subsection{The CARLA (Unreal) Approach}

As pictured in Figure 7, we have transferred the simulator study map pictured in Figure 1 into the CARLA environment. Besides this the interface to SUMO is already developed and currently being tested for different studies (https://carla.readthedocs.io/en/latest/adv_sumo/).

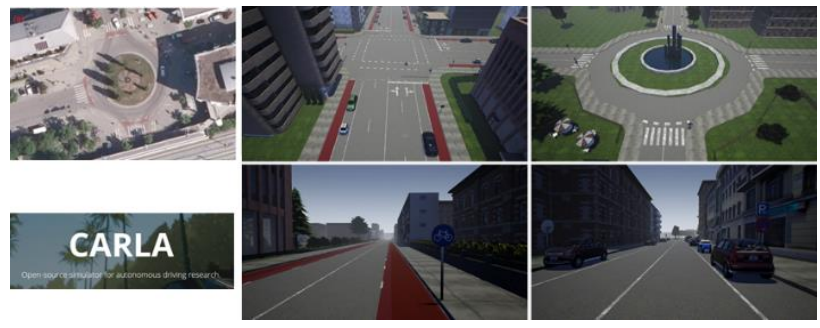

Figure 7. Overview of scenarios used for the second bicycle simulator study within the project @ CITY.

Based on Pabst (2020), there are various advantages of using open software for designing and conducting simulator studies, which is also supported by inclusions of supported open standards such as OpenDRIVE.

\subsection{The Unity3D Approach}

Besides the mentioned approaches, we are currently designing numerous simulator studies with a focus on traffic safety at intersections and bottlenecks, where cyclists are conflicting with autonomously- or manuallydriving motorized vehicles. Figure 8 show the example of a real intersection in Ingolstadt, Germany, where we introduce different scenarios with a variation in the presence of visibility obstacles for motorists and cyclists and based on this evaluate left-turning conflicts caused by the motorist. Due to the positive experience with the VR glasses of the type Oculus, we often associate the field of view with the glasses of a vulnerable road user or a passenger of an autonomously-driving car. The scenarios are then partially moving into the perspective of a pedestrian as pictured in Figure 9 with an avatar moving across different transportation infrastructure. 

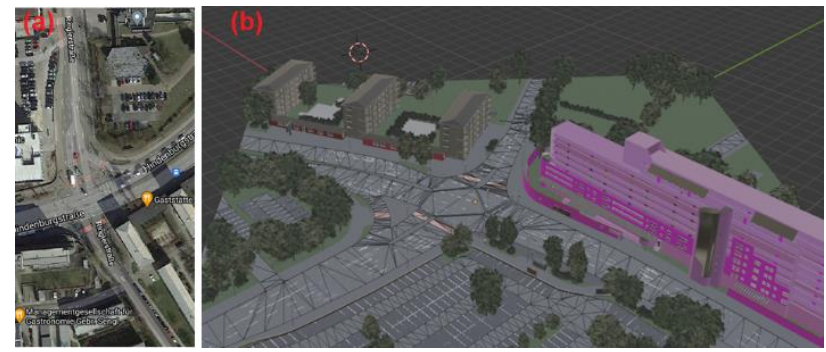

Figure 8. Designing an interconnected motorist-cyclist simulator study via adjusting infrastructural road intersection elements in a real-world example (here: an intersection in Ingolstadt, Germany) - nearby-situated parking facilities may serve as previous steering tests for both test subjects (Keler et al. 2021b).

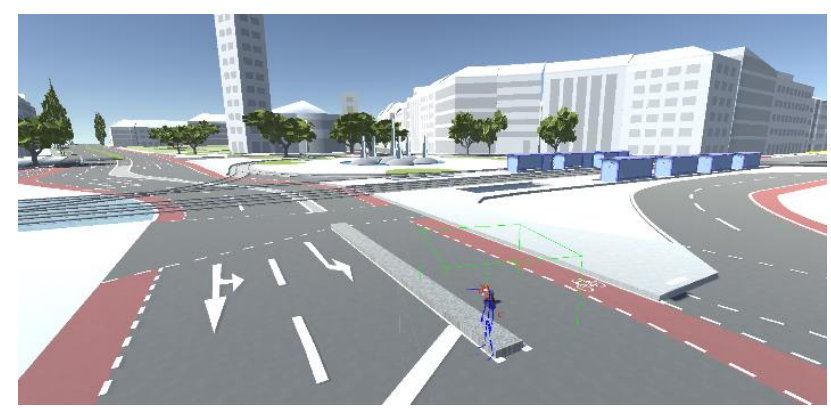

Figure 9. Validation of Markings and Bicycle Infrastructure at a depiction of the Sendlinger Tor in the City Center of Munich, Germany.

An optional component might be the conversion of the previously-converted OSM extract. This often does not deliver an acceptable result, due to often high complexities of present urban (and of course signalized) intersections. Besides the complexities in varying turning options related to specific signal programs and phases, we have multiple road users relying on specific infrastructure, such as rails for the case of the Munich tram ways.

\section{Discussion and Outlook}

The ongoing research of designing bicycle simulator maps and scenarios delivers rather practical findings, which might benefit formulating best cases. Based on the applications and research questions, we might extend the view on how data, not only geodata, can be collected from test subjects.

Future research directions might also include the semantic modelling of guidelines for urban infrastructure design that can be evaluated using the perspectives of pedestrians or cyclists. Here we see also a relation to Open geodata, OGC standard-based, formats such as CityGML, which have the advantage of the ability of having a parameterizable option of defining attribute values. In a similar way German design guidelines such as the RASt 06 or the HBS have numerous relationships that might be incorporated into interactive planning procedures based on $3 \mathrm{D}$ visualization software. Therefore, we suggest to introduce extensions for incorporating these established guidelines with an outlook of effectively present infrastructure to planners or citizens.

\section{References}

Barthauer, M. and Hafner, A. (2018). Coupling traffic and driving simulation: Taking advantage of SUMO and SILAB together. EPiC Series in Engineering, 2, 56-66.

Kaths, H., Keler, A., Grigoropoulos, G., Krämer, K. and Busch, F. (2021a). RadOnTime: Wirkungen von Restzeitanzeigen auf den Radverkehr - Steuerung des Radverkehrs. In: Radverkehrsinfrastruktur - Baustein der Verkehrswende. Deutsches Institut für Urbanistik $\mathrm{gGmbH}$.

Kaths, H., Keler, A., Grigoropoulos, G., Hosseini, S.A. and Busch, F. (2021b). RASCH - RAdSCHnellwege: Gestaltung effizienter und sicherer Infrastruktur - Schnell zum Ziel auf dem Radweg von morgen. In: Radverkehrsinfrastruktur - Baustein der Verkehrswende. Deutsches Institut für Urbanistik gGmbH.

Kaths, J. and Krause, S. (2016). Integrated simulation of microscopic traffic flow and vehicle dynamics. In IPG Apply \& Innovate 2016.

Keler, A., Grigoropoulos, G., Kaths, H., Krämer, K., Kaths, J., Spangler, M. and Busch, F. (2019a). Designing maps with fictional and real road intersections for the evaluation of countdown timer displays for bicyclists. Proceedings of the ICA 2, 2019, 1-5.

Keler, A., Grigoropoulos, G. and Mussack, D. (2019b). Enriching complex road intersections from OSM with traffic-related behavioral information. In Proceedings of the ICA 2, 2019, 1-3.

Keler, A., Mansour, M., Malcolm, P., Grigoropoulos, G., Busch, F., Bogenberger, K. (2021a) An Interconnected Motorist-Cyclist Simulator Study for Observing Communication at a Static Bottleneck - First Insights. In: DSC 2021 - 20th Driving Simulation \& Virtual Reality Conference. Munich, Germany.

Keler, A., Denk, F., Brunner, P., Grigoropoulos, G., Malcolm, P., Bogenberger, K. (2021b) Varying Bicycle Infrastructures - An Interconnected Simulator Study for Inspecting Motorist-Cyclist Conflicts. In: DSC 2021 20th Driving Simulation \& Virtual Reality Conference. Munich, Germany.

Malcolm, P. (2019) Analysis of Bicyclist Communication Patterns Using a Simulator Environment for AV Applications. Master Thesis. Technical University of Munich, Munich, Germany.

Malcolm, P., Grigoropoulos, G., Keler, A., Kaths, H. and Bogenberger, K. (2020). Introducing SumoNetVis: A Tool for Visualizing SUMO Networks and Trajectory Data. In: SUMO User Conference 2020 - From Traffic Flow to Mobility Modeling.

Pabst, P. (2020). Conception and implementation of an interface between microscopic traffic flow simulation and driving and sensor simulation. Master Thesis. Technical University of Munich, Munich, Germany. 